\title{
CONSTRUCTION AND PROPERTIES OF THE CONLEY INDEX
}

\author{
MARIAN MROZEK \\ Institute of Computer Science, Jagiellonian University \\ 30-072 Kraków, Poland \\ E-mail:mrozek@ii.uj.edu.pl
}

1. Introduction. Let $(X, d)$ be a given locally compact metric space and let

$$
\pi: X \times T \rightarrow X
$$

be a dynamical system on $X$ with continuous time $(T=\mathbb{R})$ or discrete time $(T=\mathbb{Z})$. For $t \in T$ let

$$
\pi_{t}: X \ni x \rightarrow \pi(x, t) \in X
$$

denote the $t$-translation map. Let $f:=\pi_{1}$. Note that $\pi(x, n)=f^{n}(x)$ for $n \in \mathbb{Z}$. Thus in the discrete case the map $\pi$ may be reconstructed from $f$. Therefore in the sequel we will identify a discrete dynamical system $\pi$ with its 1-translation map $f$. For $A \subset \mathbb{Z}$ we will use the notation $f^{A}(x):=\left\{f^{n}(x) \mid n \in A\right\}$.

Let $N \subset X$ be a compact subset. The maximal invariant subset of $N$ is defined by

$$
\operatorname{Inv}(N, \pi):=\operatorname{Inv} N:=\left\{x \in N \mid \forall t \in T \pi_{t}(x) \in N\right\} .
$$

We say that $S \subset X$ is invariant if Inv $S=S$. A compact set $N \subset X$ is called an isolating neighbourhood if Inv $N \subset \operatorname{int} N$. The set $S$ which admits an isolating neighbourhood $N$ such that $S=\operatorname{Inv} N$ is called an isolated invariant set. Proving the existence of compact invariant sets as well as understanding their structure and behaviour under perturbation are important goals of the theory of dynamical systems. An important special case is an isolated invariant set. Charles Conley (see [1]) showed that the properties of an isolated invariant set may be studied by investigating the isolating neighbourhood. The advantage is the fact that isolating neighbourhoods are easier to locate and understand. Conley showed that every isolating neighbourhood $N$ carries some information which depends only on the isolated invariant set $S$ in $N$. The information may be used to derive certain

1991 Mathematics Subject Classification: Primary 54H20; Secondary 58F35, 34C35.

Supported by KBN, Grant 2 P03A 02912.

The paper is in final form and no version of it will be published elsewhere. 
properties of $S$ and may be computed from the topology of certain subsets of $N$. Depending on the particular construction the information may take the form of a certain group, algebra, homotopy type of a space or just an object in an abstract category. Regardless of its particular form the information is referred to as the Conley index.

In this paper we will present a concise but relatively general construction of the Conley index. In the sequel we restrict our attention to discrete dynamical systems. This is not an essential restriction: as was shown in [3] the flow case may be reduced to the discrete case by studying iterates of the $t$-translation map of the flow for any $t \neq 0$.

An isolating neighbourhood $N$ always admits an index pair, i.e. a pair $P=\left(P_{1}, P_{2}\right)$ of compact subsets of $N$ such that the following three conditions are satisfied

(i) positive invariance relative to $N$ : $x \in P_{i}, f(x) \in N \Rightarrow f(x) \in P_{i}, \quad i=1,2$

(ii) exit set: $x \in P_{1}, f(x) \notin N \Rightarrow x \in P_{2}$

(iii) isolation: $\operatorname{Inv} N \subset \operatorname{int}\left(P_{1} \backslash P_{2}\right)$.

In fact one can prove a slightly more general result:

THEOREM 1.1. Let $N$ be an isolating neighbourhood for $f$ and $W$ a neighbourhood of Inv $N$. Then there exists an index pair $P$ for $N$ with $P_{1} \backslash P_{2} \subset W$.

We will present a proof of this theorem in Section 3.

As we already mentioned Conley observed that although there are many different index pairs for a given isolating neighbourhood, they do contain some common information. In the case of a flow the common information is the homotopy type of the quotient space $P_{1} / P_{2}$, where the quotient space $P_{1} / P_{2}$ is obtained as follows. First we take a universal point $* \notin P_{1}$ and introduce an equivalence relation $\equiv_{P}$ in $P_{1} \cup\{*\}$ given by

$$
x \equiv_{P} y \text { iff } x=y \text { or } x, y \in P_{2} \cup\{*\} .
$$

Then we take the equivalence classes of $\equiv_{P}$ as elements in $P_{1} / P_{2}$ and define the open sets in $P_{1} / P_{2}$ as sets whose counter image in the quotient map is open in $P_{1} \cup\{*\}$. In the sequel the equivalence class of $x \in P_{1} \cup\{*\}$ in the relation $\equiv_{P}$ will be denoted by $[x]_{P}$ and the space $P_{1} / P_{2}$ will be treated as a pointed space with the base point being $[*]_{P}$.

In the discrete case the extraction of the common information is slightly more complicated. First we need to observe that for every index pair $P$ there is an induced map on the quotient space $P_{1} / P_{2}$ defined by

$$
f_{P}\left([x]_{P}\right):= \begin{cases}{[f(x)]_{P}} & \text { if } x \in f^{-1}\left(P_{1}\right) \\ {[*]_{P}} & \text { otherwise. }\end{cases}
$$

It is an easy exercise to verify that $f_{P}$ is continuous and $f_{P}\left([*]_{P}\right)=[*]_{P}$. It turns out that if $f$ comes from a flow then $f_{P}$ is homotopic to the identity on $P_{1} / P_{2}$ (see [3]). This is why we can disregard $f_{P}$ in the case of a flow. In the discrete case the common information may be extracted from $\left(P_{1} / P_{2}, f_{P}\right)$. This requires introducing the category of endomorphisms.

Let $\mathcal{E}$ be a category. We define the category of endomorphisms of $\mathcal{E}$, denoted by $\operatorname{Endo}(\mathcal{E})$ as follows: the objects of $\operatorname{Endo}(\mathcal{E})$ are pairs $(A, a)$, where $A \in \mathcal{E}$ and $a \in \mathcal{E}(A, A)$ is an endomorphism of $A$. The set of morphisms from $(A, a) \in \mathcal{E}$ to $(B, b) \in \mathcal{E}$ is the 
subset of $\mathcal{E}(A, B)$ consisting of exactly those morphisms $\varphi \in \mathcal{E}(A, B)$ for which $b \varphi=\varphi a$. We write $\varphi:(A, a) \rightarrow(B, b)$ to denote that $\varphi$ is a morphism from $(A, a)$ to $(B, b)$ in $\operatorname{Endo}(\mathcal{E})$. Note that in particular $a:(A, a) \rightarrow(A, a)$ is a morphism in $\operatorname{Endo}(\mathcal{E})$.

Let $\mathcal{C}$ be another category and $L: \operatorname{Endo}(\mathcal{E}) \rightarrow \mathcal{C}$ be a functor. We say that $L$ is normal if $L(a)$ is an isomorphism in $\mathcal{C}$ for any endomorphism $a: A \rightarrow A$ in $\mathcal{E}$. The reason we are interested in normal functors is the following theorem.

Theorem 1.2. Assume $L: \operatorname{Endo}(\mathcal{E}) \rightarrow \mathcal{C}$ is a normal functor and $\varphi:(A, a) \rightarrow(B, b)$, $\psi:(B, b) \rightarrow(A, a)$ are such that $a=\varphi \psi, b=\psi \varphi$. Then we have the commutative diagram

$$
\begin{aligned}
& L(A, a) \stackrel{L(a)}{\longrightarrow} L(A, a) \\
& \downarrow L(\varphi) \nearrow L(\psi) \downarrow L(\varphi) \\
& L(B, b) \underset{L(b)}{\longrightarrow} L(B, b)
\end{aligned}
$$

in $\mathcal{C}$, in which all morphisms are isomorphisms.

Proof. The commutativity of the diagram is obvious. The equality $L(\psi) L(\varphi)=L(a)$ implies

$$
\left(L(a)^{-1} L(\psi)\right) L(\varphi)=\operatorname{id}_{L(A, a)}
$$

and $L(\varphi) L(\psi)=L(b)$ implies

$$
L(\varphi)\left(L(\psi) L(b)^{-1}\right)=\operatorname{id}_{L(B, b)} .
$$

Hence $L(\varphi)$ is an isomorphism. The proof for $L(\psi)$ is similar.

It turns out that normal functors are useful in extracting from an isolating neighborhood information dependent only on the isolated invariant set inside. The first step

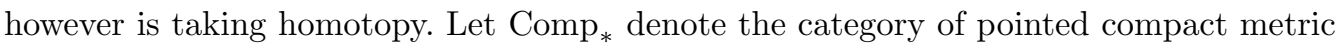
spaces and let $\mathrm{HComp}_{*}$ denote the homotopy category of pointed compact metric spaces. We recall that objects in both categories are pointed compact spaces (compact spaces with a selected base point) and morphisms in Comp ${ }_{*}$ are continuous maps preserving the base point whereas morphisms in $\mathrm{HComp}_{*}$ are homotopy classes of such maps. There is also the homotopy functor Htp : $\mathrm{Comp}_{*} \rightarrow \mathrm{HComp}_{*}$ which fixes objects and sends every base point preserving continuous map $\varphi: X \rightarrow Y$ to its homotopy class denoted by $[\varphi]$.

Let $L: \operatorname{Endo}\left(\mathrm{HComp}_{*}\right) \rightarrow \mathcal{C}$ be a normal functor. (We will show in the next section that every category admits a normal functor.)

THEOREM 1.3. Let $S$ be an isolated invariant set, $N$ be an isolating neighbourhood for $S$ and $P$ be an index pair in $N$. Then $C(S, f):=C(N, f):=L\left(P_{1} / P_{2},\left[f_{P}\right]\right)$ is independent of the choice of $N$ and an index pair $P$ in $N$.

The object $C(S, f)$ given by the above theorem is called the Conley index of $S$.

2. The Szymczak functor. In order to construct a category $\mathcal{C}$ and a normal functor $L: \operatorname{Endo}(\mathcal{E}) \rightarrow \mathcal{C}$ we want to modify the category $\operatorname{Endo}(\mathcal{E})$ in such a way that every morphism $e:(E, e) \rightarrow(E, e)$ becomes an isomorphism. Thus let's speculate and see what happens if we add an artificial inverse of $e$ denoted by $\bar{e}$. If $\varphi:(E, e) \rightarrow(F, f)$ is a 
morphism in $\operatorname{Endo}(\mathcal{E})$, then the equality $\varphi \circ e=f \circ \varphi$ implies that also

$$
\bar{f} \circ \varphi=\varphi \circ \bar{e} \text {. }
$$

To preserve the category structure for every sequence $\left\{\varphi_{i}\right\}_{i=1, n}$ of morphisms $\varphi_{i}$ : $\left(E_{i-1}, e_{i-1}\right) \rightarrow\left(E_{i}, e_{i}\right)$ and any sequence $\left\{k_{i}\right\}_{i=0, n}$ of natural numbers we need to add the composition

$$
\bar{e}_{n}^{k_{n}} \circ \varphi_{n} \circ \bar{e}_{n-1}^{k_{n-1}} \circ \cdots \circ \varphi_{1} \circ \bar{e}_{0}^{k_{0}} .
$$

However it follows from (1) that the above composition equals

$$
\varphi_{n} \circ \varphi_{n-1} \circ \cdots \varphi_{1} \circ \bar{e}_{0}^{n_{k}+n_{k-1}+\ldots+n_{0}} .
$$

Thus what we actually need to add are all morphisms of the form

$$
\varphi \circ \bar{e}^{m}
$$

for any morphism $\varphi:(E, e) \rightarrow(F, f)$ and any natural number $m$. Notice that the morphism $\bar{e}$ is uniquely determined by the morphism $\varphi$. Therefore it is natural to identify the morphism (2) with the pair $(\varphi, m)$. The formula (2) implies that the definition of the composition of the added morphisms should read

$$
(\psi, k) \circ(\varphi, l):=(\psi \circ \varphi, k+l),
$$

where $\varphi:(E, e) \rightarrow(F, f), \psi:(F, f) \rightarrow(G, g)$ and $k, l \in \mathbb{N}$. However there is one problem with the above definition: it may happen that for $\varphi, \varphi^{\prime}:(E, e) \rightarrow(F, f)$ we have:

$$
\varphi \circ e^{m^{\prime}+k}=\varphi^{\prime} \circ e^{m+k}
$$

for some $m, m^{\prime}, k \in \mathbb{N}$. Then multiplying formula (3) on the right hand side by $\bar{e}^{m+m^{\prime}+k}$ we obtain

$$
\varphi \circ \bar{e}^{m}=\varphi^{\prime} \circ \bar{e}^{m^{\prime}}
$$

i.e.

$$
(\varphi, m)=\left(\varphi^{\prime}, m^{\prime}\right)
$$

Thus we need to introduce an equivalence relation. Namely we write

$$
(\varphi, m) \equiv\left(\varphi, m^{\prime}\right)
$$

whenever there exists a $k \in \mathbb{N}$ such that the condition (3) is satisfied. One easily verifies that this indeed defines an equivalence relation in $\operatorname{Endo}(\mathcal{E})((E, e),(F, f))$. The Szymczak category of endomorphisms of $\mathcal{E}, \operatorname{Szym}(\mathcal{E})$ can now be defined formally as follows. The objects of $\operatorname{Szym}(\mathcal{E})$ are the same as in $\operatorname{Endo}(\mathcal{E})$. The morphisms in $\operatorname{Szym}(\mathcal{E})((E, e),(F, f))$ are the equivalence classes of the relation (5). One easily checks that the definition of composition carries over to the equivalence classes. The identity morphism on $(E, e) \in$ $\operatorname{Szym}(\mathcal{E})$ is $\left[\operatorname{id}_{E}, 0\right]$. There is a functor $\operatorname{Szym}: \operatorname{Endo}(\mathcal{E}) \rightarrow \operatorname{Szym}(\mathcal{E})$ which fixes objects and sends a morphism $\varphi:(E, e) \rightarrow(F, f)$ to the equivalence class $[\varphi, 0]$. In general it may happen that $\operatorname{Szym}(\varphi)=\operatorname{Szym}\left(\varphi^{\prime}\right)$ even if $\varphi \neq \varphi^{\prime}$. Nevertheless it is convenient to write just $\varphi$ to denote $\operatorname{Szym}(\varphi)$ whenever it is clear from the context in which category we work. Now we can formally define the inverse of $e:(E, e) \rightarrow(E, e)$ in $\operatorname{Szym}(\mathcal{E})$ by

$$
\bar{e}:=\left[\operatorname{id}_{E}, 1\right] .
$$


Indeed, we have

$$
e \circ \bar{e}=[e, 0] \circ\left[\operatorname{id}_{E}, 1\right]=[e, 1]=\left[\operatorname{id}_{E}, 0\right]=\operatorname{id}_{(E, e)}
$$

which shows that $\bar{e}$ is an inverse of $e$. We can also write the abstract morphism $[\varphi, n]$ in terms of $\bar{e}$ as

$$
[\varphi, n]=[\varphi, 0] \circ\left[\operatorname{id}_{E}, 1\right]^{n}=\varphi \circ \bar{e}^{n} .
$$

Obviously $\operatorname{Szym}(e)$ is invertible in $\operatorname{Szym}(\mathcal{E})$, therefore $\operatorname{Szym}$ is a normal functor. Actually this is the most general normal functor. The reader may easily show that any other normal functor $L: \operatorname{Endo}(\mathcal{E}) \rightarrow \mathcal{C}$ admits a unique functor $L^{\prime}: \operatorname{Szym}(\mathcal{E}) \rightarrow \mathcal{C}$ such that $L=L^{\prime} \circ$ Szym. The construction of the Szymczak category and the Szymczak functor is due to Szymczak [8].

3. Construction of index pairs. Construction of index pairs is probably the most technical part of the Conley index theory. There are several different proofs available (see [1], [5], [6], [7]). We will present here a proof which goes back to some early constructions. First we recall the following straightforward proposition.

Proposition 3.1. Assume $N \subset X$ is compact. Then the set $\operatorname{Inv} N$ is compact and so are the sets

$$
\begin{aligned}
& \operatorname{Inv}^{-} N:=\left\{x \in N \mid \forall n \in \mathbb{Z}^{-} f^{n}(x) \in N\right\}, \\
& \operatorname{Inv}^{+} N:=\left\{x \in N \mid \forall n \in \mathbb{Z}^{+} f^{n}(x) \in N\right\} .
\end{aligned}
$$

To guide the reader's intuition, we sketch the proof first, developing the necessary lemmas. The idea is to take as the first approximation for $P_{1}$ a compact neighbourhood $A$ of $\mathrm{Inv}^{-} N$ and as the first approximation of $P_{2}$ the set $P_{1} \backslash U$, where $U$ is an open neighbourhood of $\mathrm{Inv}^{+} N$. Such a pair does meet the isolation condition but obviously it need not meet the relative positive invariance condition. Therefore we enlarge the sets by following them along trajectories as long as they stay in $N$. Formally this is done as follows. For $x \in N$ we put

$$
f_{N}^{+}(x):=\left\{y \in N \mid \exists j \in \mathbb{Z} y=f^{j}(x) \text { and } f^{[0, j]}(x) \subset N\right\} .
$$

This actually defines a multivalued map. We recall that a multivalued map is a map $F: X \rightarrow \mathcal{P}(Y)$, where $\mathcal{P}(Y)$ stands for the family of all subsets of $Y$. If $F: X \rightarrow \mathcal{P}(Y)$ is a multivalued map and $A \subset X$ then the image of $A$ under $F$ is defined by $F(A):=$ $\bigcup_{x \in A} F(x)$. Thus $f_{N}^{+}: N \ni x \rightarrow f_{N}^{+}(x) \in \mathcal{P}(N)$ is a multivalued map. We put

$$
\begin{gathered}
P_{1}:=f_{N}^{+}(A), \\
P_{2}:=f_{N}^{+}\left(P_{1} \backslash U\right) .
\end{gathered}
$$

The first question now is if the modified sets are still compact. They would be compact if $f_{N}^{+}$were an upper semicontinuous map. A multivalued map $F: X \rightarrow \mathcal{P}(Y)$ is upper semicontinuous if for every $x \in X$ and every open set $U$ such that $F(x) \subset U$ there exists an open neighbourhood $V$ of $x$ such that $y \in V$ implies $F(y) \subset U$. A well known fact is that the image of a compact set under a compact valued upper semicontinuous map is compact. 
Unfortunately $f_{N}^{+}$need not be upper semicontinuous. However, for every fixed $n \in \mathbb{N}$ the map $f_{N, n}: N \rightarrow \mathcal{P}(N)$ given by

$$
f_{N, n}(x):=\left\{y \in N \mid \exists j \in[0, n] y=f^{j}(x) \text { and } f^{[0, j]}(x) \subset N\right\}
$$

is upper semicontinuous:

Proposition 3.2. The map $f_{N, n}: N \rightarrow \mathcal{P}(N)$ is upper semicontinuous for any $n \in \mathbb{N}$.

Proof. Suppose that $f_{N, n}$ is not upper semicontinuous. Then there exists a point $x \in$ $N$ and an open subset $U$ of $N$ satisfying $f_{N, n}(x) \subset U$ and a convergent sequence $x_{k} \rightarrow x$, $\left\{x_{k}\right\} \subset N$ with $f_{N, n}\left(x_{k}\right) \not \subset U$. Consequently for each $k$, there exists an $m_{k} \in[0, n]$ such that $f^{m_{k}}\left(x_{k}\right) \in N \backslash U$. Passing to a subsequence we may assume that $m_{k}=m \in[0, n]$ for all $k$. It follows that $f^{m}(x) \notin U$, a contradiction.

One can easily see that

$$
f_{N}^{+}(x):=\bigcup_{n \in \mathbb{Z}^{+}} f_{N, n}(x),
$$

unfortunately, upper semicontinuity does not carry over to infinite unions. However, if $x \notin \mathrm{Inv}^{+} N$, then the above union is obviously finite and one can expect that this property carries over to compact sets. Indeed, we have the following lemma.

Lemma 3.3. Let $K \subset N$ be a compact subset of $X$ such that $K \cap \operatorname{Inv}^{+} N=\emptyset$. Then

(a) $f_{N, n}(K)=\emptyset$ for all but finitely many $n \in \mathbb{N}$,

(b) The mapping $f_{N}^{+}$restricted to $K$ is upper semicontinuous,

(c) $f_{N}^{+}(K) \cap \operatorname{Inv}^{+} N=\emptyset$.

Proof. Since $x \in K$ implies $x \notin \mathrm{Inv}^{+} N$, for each $x \in K$ there exists an $n_{x} \in \mathbb{Z}^{+}$such that $f_{N, n_{x}}(x)=\emptyset$. Since $f_{N, n_{x}}$ is upper semicontinuous, there exists a neighbourhood $V_{x}$ of $x$ such that $f_{N, n_{x}}\left(V_{x}\right)=\emptyset$. Let $\left\{V_{x_{1}}, \ldots V_{x_{k}}\right\}$ be a finite covering of $K$. Put

$$
m:=\max \left\{n_{x_{i}} \mid i=1,2, \ldots k\right\} .
$$

Then $f_{N, n}(K)=\emptyset$ for all $n \geq m$. This proves (a). Property (b) follows from (a) and Proposition 3.2 since the union of finitely many upper semicontinuous maps is upper semicontinuous. Property (c) is straightforward.

Returning to our construction we see that $P_{2}=f_{N}^{+}\left(P_{1} \backslash U\right)$ is compact, because $K:=P_{1} \backslash U$ satisfies the assumptions of the above lemma. Concerning $P_{1}$, we have the following lemma.

Lemma 3.4. If $\mathrm{Inv}^{-} N \subset A \subset N$ and $A$ is compact then $f_{N}^{+}(A)$ is compact.

Proof. It is sufficient to show that $f_{N}^{+}(A)$ is closed. Let $\left\{y_{k}\right\}$ be a sequence of points in $f_{N}^{+}(A), y_{k} \rightarrow y \in N$. We need to show that $y \in f_{N}^{+}(A)$. Let $x_{k} \in A, n_{k} \in \mathbb{Z}^{+}$be such that $y_{k}=f^{n_{k}}\left(x_{k}\right)$ and $f^{\left[0, n_{k}\right]}\left(x_{k}\right) \subset N$. We will consider first the case when $\left\{n_{k}\right\}$ is bounded. Then, passing to a subsequence if necessary, we may assume that $n_{k}=n$ for all $k$. Since $N$ and $A$ are compact, we conclude that $y=f^{n}(x)$ and $f^{[0, n]}(x) \subset N$, i.e. $y \in f_{N}^{+}(A)$. Hence it remains to consider the case when $\left\{n_{k}\right\}$ is unbounded. Then, passing to a subsequence, we may assume that $\left\{n_{k}\right\}$ is increasing and, by restricting the interval 
$\left[0, n_{k}\right]$, that $n_{k}=k$. It follows that for any $m \in \mathbb{Z}^{-}$and almost all $k \in \mathbb{Z}^{+} f^{m}\left(y_{k}\right) \in N$, hence also $f^{m}(y) \in N$. Thus $y \in \mathrm{Inv}^{-} N \subset A$.

Thus we have a pair $\left(P_{1}, P_{2}\right)$ of compact sets which are positively invariant with respect to $N$. The exit set property is equivalent to showing that $P_{1} \backslash P_{2} \subset f^{-1}(N)$. Since Inv $N$ is invariant under $f$, one can expect that points close to Inv $N$ cannot be mapped by $f$ outside of $N$. Thus the exit set property will be guaranteed if we choose $A$ and $U$ in such a way that $P_{1} \backslash P_{2}$ is close to Inv $N$. Actually, this stronger postulate is a part of the assertion of Theorem 1.1 anyway. To fulfill it, given a neighbourhood $W$ of Inv $N$, we take $U$ an open neighbourhood of $\operatorname{Inv}^{+} N$ and $V$ an open neighbourhood of $\operatorname{Inv}^{-} N$ such that $U \cap V \subset W$. This is always possible, because Inv $N=\operatorname{Inv}^{+} N \cap \operatorname{Inv}^{-} N$. Then $P_{1} \backslash U \subset f_{N}^{+}\left(P_{1} \backslash U\right)=P_{2}$, which implies that $P_{1} \backslash P_{2} \subset U$. To guarantee that $P_{1} \backslash P_{2} \subset W$ it is sufficient now to show that one can choose $A$ in such a way that $P_{1}=f_{N}^{+}(A) \subset V$. This is the contents of the following lemma.

Lemma 3.5. For any neighbourhood $V$ of $\mathrm{Inv}^{-} N$ there exists a compact neighbourhood $A$ of $\operatorname{Inv}^{-} N$ such that $f_{N}^{+}(A) \subset V$.

Proof. Similarly to the map $f_{N, n}$ for any $n \in \mathbb{N}$ we define the map $f_{N,-n}: N \rightarrow \mathcal{P}(N)$ given by

$$
f_{N,-n}(x):=\left\{y \in N \mid \exists j \in[-n, 0] y=f^{j}(x) \text { and } f^{[j, 0]}(x) \subset N\right\}
$$

The set $K:=N \backslash V$ is disjoint from $\mathrm{Inv}^{-} N$. An argument dual to the proof of Lemma 3.3 shows that there exists an $m \in \mathbb{Z}^{+}$satisfying

$$
f_{N,-m}(N \backslash V)=\emptyset .
$$

Basically the same proof as in Proposition 3.2 shows that $f_{N,-m}$ is upper semicontinuous. Therefore one can find for every $x \in \mathrm{Inv}^{-} N$ a compact neighbourhood $V_{x}$ of $x$ such that $f_{N,-m}\left(V_{x}\right) \subset V$. Let $\left\{V_{x_{1}}, \ldots V_{x_{k}}\right\}$ be a finite covering of $\operatorname{Inv}^{-} N$. Put $A:=\bigcup_{i=1}^{k} V_{x_{i}}$. Then $A$ is a compact neighbourhood of $\operatorname{Inv}^{-} N$ such that $f_{N,-m}(A) \subset V$. It remains to show that $f_{N}^{+}(A) \subset V$. Indeed, let $y \in f_{N}^{+}(A)$. Then there exists an $n>0$ and an $x \in A$ such that $y \in f_{N, n}(x)$. If $n \leq m$, we are done. If $n>m$, we note that $x \in f_{N,-n}(y)$, hence $f_{N,-m}(y) \neq \emptyset$ and (6) implies that $y \in V$.

We are now ready to summarize the proof of the existence of index pairs.

Proof of Theorem 1.1. Since $\operatorname{Inv} N=f(\operatorname{Inv} N)$ is a compact subset of $\operatorname{int} N$, we may assume that $W \cup f(W) \subset \operatorname{int} N$. Let $A$ be given for $V$ by Lemma 3.5. We define

$$
P_{1}:=f_{N}^{+}(A), P_{2}:=f_{N}^{+}\left(P_{1} \backslash U\right) .
$$

Then $P_{1} \subset V$ and $P_{1} \backslash U \subset P_{2}$ which implies that $P_{1} \backslash P_{2} \subset U$. Therefore $P_{1} \backslash P_{2} \subset$ $U \cap V \subset W$. We verify that $\left(P_{1}, P_{2}\right)$ is an index pair. $P_{1}$ is compact by Lemma 3.4 and $P_{2}$ is compact by Lemma 3.3 (b), since $P_{1} \backslash U$ is compact and the image of a compact set under an upper semicontinuous compact valued map is compact. Obviously, $P_{2} \subset$ $f_{N}^{+}\left(P_{1}\right) \subset P_{1}$. Positive invariance of $P_{1}$ and $P_{2}$ relative to $N$ is obvious. Since $P_{1} \backslash P_{2} \subset W$ and $f(W) \subset \operatorname{int} N$, we see that the exit set property is satisfied. In order to verify the isolation property, observe that $P_{1}$ is a neighbourhood of $\operatorname{Inv}^{-} N$ and, by Lemma 3.3 (c), 
$N \backslash P_{2}$ is a neighbourhood of $\operatorname{Inv}^{+} N$. Therefore $P_{1} \backslash P_{2}=P_{1} \cap\left(N \backslash P_{2}\right)$ is a neighbourhood of $\operatorname{Inv}^{-} N \cap \operatorname{Inv}^{+} N=\operatorname{Inv} N$.

4. Correctness of the definition. There are several different proofs of the correctness of the construction of the Conley index. What one has to prove is that given an isolated invariant set $S$, its two isolating neighbourhoods $N$ and $M$ and index pairs $P=\left(P_{1}, P_{2}\right), Q=\left(Q_{1}, Q_{2}\right)$ respectively in $N$ and $M$, the objects $L\left(\left[P_{1} / P_{2}\right],\left[f_{P}\right]\right)$ and $L\left(\left[Q_{1}, Q_{2}\right],\left[f_{Q}\right]\right)$ are isomorphic. One way to prove this is to construct explicitly the isomorphism (see [8]). However the explicit formula for the isomorphism is complicated and it is of no use once the proof is completed. Therefore we will choose here another approach, which follows the ideas in [4]. Again, to guide the reader's intuition, we sketch the proof first, developing the necessary lemmas.

The first observation is that it is relatively easy to write down the isomorphism if $M=N$ and the index pairs $P$ and $Q$ do not lie too far apart. To be more specific, assume that $P \subset Q$ and

$$
f\left(Q_{i}\right) \cap N \subset P_{i} \text { for } i=1,2 .
$$

Then one can define the map $f_{Q P}: Q_{1} / Q_{2} \rightarrow P_{1} / P_{2}$ by

and the map $\iota_{P Q}: P_{1} / P_{2} \rightarrow Q_{1} / Q_{2}$ by

$$
f_{Q P}\left([x]_{Q}\right):= \begin{cases}{[f(x)]_{P}} & \text { if } x \in f^{-1}(N) \\ {[*]} & \text { if } x \in Q_{2} \cup\{*\} .\end{cases}
$$

$$
\iota_{P Q}\left([x]_{P}\right):=[x]_{Q} .
$$

The reader will check easily that both maps are well defined and continuous. We have the following lemma.

Lemma 4.1. Assume that $P \subset Q$ are index pairs in $N$ such that (8) is satisfied. If $L: \operatorname{Endo}\left(\mathrm{HComp}_{*}\right) \rightarrow \mathcal{C}$ is a normal functor then $L\left(\left[\iota_{P Q}\right]\right)$ is an isomorphism in $\mathcal{C}$.

Proof. One easily verifies that the following diagram

$$
\begin{array}{ccc}
P_{1} / P_{2} & \stackrel{f_{P}}{\longrightarrow} & P_{1} / P_{2} \\
\downarrow \iota_{P Q} & \nearrow f_{Q P} & \mid \iota_{P Q} \\
Q_{1} / Q_{2} & \stackrel{f_{Q}}{\longrightarrow} & Q_{1} / Q_{2}
\end{array}
$$

commutes. Applying the homotopy functor and Theorem 1.2 we get the required conclusion.

The next step is to get rid of the assumption (8). This may be achieved by finding a sequence of index pairs $P=Q^{n} \subset Q^{n-1} \subset \cdots \subset Q^{1} \subset Q^{0}=Q$ such that any two consecutive index pairs in the sequence satisfy condition (8). The sequence may be constructed recursively by pushing forward the larger index pair into the smaller index pair with the map $f$. Unfortunately the procedure requires an extra assumption to guarantee that the construction really produces a new index pair, for instance it suffices to assume that the index pairs $P, Q$ differ in at most one coordinate. We will see later on that there is no problem in getting rid of that assumption in the next step. The following two lemmas formalize the described procedure. 
Lemma 4.2. Let $P \subset Q$ be index pairs for $N$ which differ in at most one coordinate, i.e. $P_{1}=Q_{1}$ or $P_{2}=Q_{2}$. Define a pair of sets $G(P, Q)$ by

$$
G_{i}(P, Q)=P_{i} \cup\left(f\left(Q_{i}\right) \cap N\right) \text {, for } i=1,2 .
$$

Then

(a) If $P_{i}=Q_{i}$ then $G_{i}(P, Q)=P_{i}=Q_{i}$, for $i=1,2$.

(b) $P \subset G(P, Q) \subset Q$.

(c) $G(P, Q)$ is an index pair.

(d) $f\left(Q_{i}\right) \cap N \subset G_{i}(P, Q)$, for $i=1,2$.

Proof. Property (d) is obvious and property (a) is immediate from the positive invariance of $P_{i}, Q_{i}$ relative to $N$. The first inclusion in (b) is obvious and the second is an immediate consequence of the positive invariance of $Q_{i}$ relative to $N$. It remains to prove (c). To prove the positive invariance relative to $N$ let $x \in G_{i}(P, Q)$ and $y:=$ $f(x) \in N$. If $x \in P_{i}$ then obviously $y \in G_{i}(P, Q)$. If $x \in f\left(Q_{i}\right) \cap N$ then $x \in Q_{i}$ hence $y \in f\left(Q_{i}\right) \cap N \subset G_{i}(P, Q)$. To prove the exit set property let us note that

$$
G_{1}(P, Q) \backslash G_{2}(P, Q) \subset Q_{1} \backslash G_{2}(P, Q) \subset Q_{1} \backslash P_{2}
$$

and since $P$ and $Q$ differ in at most one coordinate either $Q_{1} \backslash P_{2}=Q_{1} \backslash Q_{2} \subset f^{-1}(N)$, or $Q_{1} \backslash P_{2}=P_{1} \backslash P_{2} \subset f^{-1}(N)$. Finally observe that $\operatorname{Inv} N \subset \operatorname{int}\left(P_{1} \backslash P_{2}\right) \cap \operatorname{int}\left(Q_{1} \backslash Q_{2}\right)$ so the isolation property will follow if we verify that $\left(P_{1} \backslash P_{2}\right) \cap\left(Q_{1} \backslash Q_{2}\right) \subset G_{1}(P, Q) \backslash G_{2}(P, Q)$. Indeed, let $y \in\left(P_{1} \backslash P_{2}\right) \cap\left(Q_{1} \backslash Q_{2}\right)$. Then $y \in G_{1}(P, Q)$ and it remains to show that $y \notin f\left(Q_{2}\right) \cap N$. However if $y \in f\left(Q_{2}\right) \cap N$, then $y \in Q_{2}$, a contradiction.

Lemma 4.3. Let $P \subseteq Q$ be index pairs which differ in at most one coordinate. Then there exists a sequence of pairs

$$
P=Q^{n} \subset Q^{n-1} \subset \ldots \subset Q^{1} \subset Q^{0}=Q
$$

with the following properties

(a) If $P_{i}=Q_{i}$ then $Q_{i}^{k}=P_{i}=Q_{i}$ for all $k=1,2, \ldots n-1 ; i=1,2$.

(b) $Q^{k}$ is an index pair for all $k=1,2, \ldots n-1$.

(c) $f\left(Q_{i}^{k}\right) \cap N \subset Q_{i}^{k+1}, i=1,2 ; k=0,1, \ldots n-1$.

Proof. Let $Q^{k}$ be given by the recurrence formula $Q^{0}:=Q, Q^{k+1}:=G\left(P, Q^{k}\right), k \in$ $\mathbb{N}$. By Lemma 4.2 and induction on $k,\left\{Q^{k}\right\}$ is a decreasing sequence of index pairs containing $P$ and satisfying (a), (b) and (c) for all $k \in \mathbb{Z}$. It remains to show that $Q^{n}=P$ for some $n$. Indeed, suppose that the inclusion $P \subset Q^{k}$ is strict for all $k \in \mathbb{N}$, i.e. if $i \in\{1,2\}$ is such that $P_{i} \neq Q_{i}$ then $Q_{i}^{k} \backslash P_{i} \neq \emptyset$. Fix a $k \in \mathbb{N}$ and choose an $x_{k} \in Q_{i}^{k} \backslash P_{i}$. We will show by induction in $j$ that

$$
f^{-j}\left(x_{k}\right) \in Q_{i}^{k-j} \backslash P_{i} \text { for } j=0,1, \ldots, k .
$$

The assertion is obvious for $j=0$. If $f^{-j}\left(x_{k}\right) \in Q_{i}^{k-j} \backslash P_{i}$, then $f^{-j}\left(x_{k}\right) \in f\left(Q_{i}^{k-j-1}\right) \cap$ $N$, i.e. $f^{-(j+1)}\left(x_{k}\right) \in Q_{i}^{k-(j+1)}$. We cannot have $f^{-(j+1)}\left(x_{k}\right) \in P_{i}$, because otherwise $f^{-j}\left(x_{k}\right) \in P_{i}$. Thus the assertion (10) is proved. Put $u_{k}:=f^{-k}\left(x_{2 k}\right)$. It follows from (10) that

$$
f^{[-k, k]}\left(u_{k}\right) \subset Q_{i} \backslash P_{i} \subset Q_{i} \backslash \operatorname{int} P_{i}
$$


Taking a subsequence we may assume that $u_{n} \rightarrow u \in Q_{i} \backslash$ int $P_{i}$ and it follows from (11) that $f^{j}(u) \in Q_{i} \backslash \operatorname{int} P_{i}$ for $j \in \mathbb{Z}$, i.e. $u \in \operatorname{Inv}\left(Q_{i} \backslash \operatorname{int} P_{i}\right)$.

If $i=1$ we get $u \in \operatorname{Inv}\left(Q_{1} \backslash \operatorname{int} P_{1}\right) \subset \operatorname{Inv} N \subset \operatorname{int}\left(P_{1} \backslash P_{2}\right) \subset \operatorname{int} P_{1}$, a contradiction. If $i=2$, we get $u \in \operatorname{Inv}\left(Q_{2} \backslash \operatorname{int} P_{2}\right) \subset \operatorname{Inv} Q_{2}$. On the other hand $\operatorname{Inv} Q_{2} \subset \operatorname{Inv} N \subset$ $\operatorname{int}\left(Q_{1} \backslash Q_{2}\right) \subset Q_{1} \backslash Q_{2}$, which implies that Inv $Q_{2}=\emptyset$, a contradiction again.

The following proposition is a straightforward observation which will let us get rid of the assumption that $P$ and $Q$ differ in at most one coordinate.

Proposition 4.4. If $P \subseteq Q$ are index pairs for $N$, then so are $\left(P_{1}, P_{1} \cap Q_{2}\right)$ and $\left(P_{1} \cup Q_{2}, Q_{2}\right)$.

In order to consider index pairs which are not necessarily included in one another, we need the following lemma.

Lemma 4.5. If $P$ and $Q$ are index pairs for $N$, then so is $P \cap Q$.

Proof. Positive invariance relative to $N$ and exit set property are obvious. To prove the isolation property let us note that $\operatorname{int}\left(P_{1} \backslash P_{2}\right) \cap \operatorname{int}\left(Q_{1} \backslash Q_{2}\right) \subset \operatorname{int}\left(P_{1} \cap Q_{1} \backslash\left(P_{2} \cup Q_{2}\right)\right) \subset$ $\operatorname{int}\left(P_{1} \cap Q_{1} \backslash P_{2} \cap Q_{2}\right)$.

Finally, we will also need the following straightforward proposition.

Proposition 4.6. Assume $P \subset Q$ are compact pairs such that $P_{1} \backslash P_{2}=Q_{1} \backslash Q_{2}$. Then $P_{1} / P_{2}$ and $Q_{1} / Q_{2}$ are homeomorphic.

We are now ready to present the proof of Theorem 1.3.

Proof of Theorem 1.3. We need to show that if $M$ and $N$ are two isolating neighbourhoods of $S, P$ an index pair for $N$ and $Q$ an index pair for $M$ then $L\left(P_{1} / P_{2},\left[f_{P}\right]\right)$ and $L\left(Q_{1} / Q_{2},\left[f_{Q}\right]\right)$ are isomorphic in $\mathcal{C}$. The proof will be given in several steps.

STEP 1. We begin with the following special case

(i) $\quad M=N$,

(ii) $P \subset Q$,

(iii) $P$ and $Q$ differ in at most one coordinate,

(iv) $f\left(Q_{i}\right) \cap N \subset P_{i}$, for $i=1,2$.

The conclusion in this case follows immediately from Lemma 4.1.

SteP 2. We lift the assumption (iv). By Lemma 4.3 we can take a sequence $\left\{Q^{k}\right\}_{k=0,1, \ldots, n}$ of index pairs such that $Q_{0}=Q, Q_{n}=P$ and the pair of index pairs $Q^{k+1} \subseteq Q^{k}$ satisfies the assumptions (i) through (iv). The conclusion follows.

STEP 3. We lift the assumption (iii). Put $R_{1}:=P_{1} \cup Q_{2}, R_{2}:=P_{1} \cap Q_{2}$. By Proposition $4.4,\left(P_{1}, R_{2}\right)$ and $\left(R_{1}, Q_{2}\right)$ are index pairs. We have the commutative diagram of inclusions

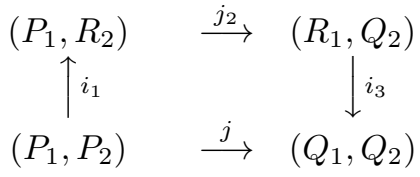


It is clear that the pair of index pairs $\left(P_{1}, P_{2}\right) \subset\left(P_{1}, R_{2}\right)$ satisfies the assumption (iii) and so does $\left(R_{1}, Q_{2}\right) \subset\left(Q_{1}, Q_{2}\right)$, so by Step 2, the inclusions $i_{1}$ and $i_{3}$ induce isomorphisms in $\mathcal{C}$. Since $P_{1} \backslash R_{2}=P_{1} \backslash Q_{2}=R_{1} \backslash Q_{2}$, it follows from Proposition 4.6 that the inclusion $j_{2}$ induces an isomorphism in $\mathcal{C}$.

SteP 4. Now, only (i) is assumed. By Lemma 4.5 $P \cap Q$ is an index pair, hence the conclusion of Step 3 can be applied to pairs $P \cap Q \subset P$ and $P \cap Q \subset Q$.

STEP 5 . If $M \neq N$, one may always assume that $M \subset N$ since otherwise $M \cap N$ can be considered which is also an isolating neighbourhood of $S$. By Step 4, it is sufficient to show the existence of one index pair $P$ for $N$ and one index pair $Q$ for $M$ such that $L\left(P_{1} / P_{2},\left[f_{P}\right]\right)$ and $L\left(Q_{1} / Q_{2},\left[f_{Q}\right]\right)$ are isomorphic.

By Theorem 1.1 there exists an index pair $P$ for $N$ such that $P_{1} \backslash P_{2} \subset \operatorname{int} M \cap$ $f^{-1}$ (int $\left.M\right)$. It is easily verified that $Q:=\left(M \cap P_{1}, M \cap P_{2}\right)$ is an index pair for $M$. Since $Q_{1} \backslash Q_{2}=M \cap\left(P_{1} \backslash P_{2}\right)=P_{1} \backslash P_{2}$, the inclusion $Q \subset P$ induces an isomorphism in $\mathcal{C}$ by Proposition 4.6.

5. Properties of the Conley index. The Conley index has several properties which facilitate its computation.

THEOREM 5.1 (Ważewski property). If $N$ is an isolating neighbourhood with respect to $f$ and $\operatorname{Con}(N, f) \neq \operatorname{Con}(\emptyset, f)$ then $\operatorname{Inv} N$ is non-empty.

Proof. If Inv $N=\emptyset$, then $(\emptyset, \emptyset)$ is an index pair for $N$. It follows that $\operatorname{Con}(N, f)=$ $\operatorname{Con}(\emptyset, f)$.

The following two properties are studied in detail in [2].

THEOREM 5.2 (Stability property). If $N$ is an isolating neighbourhood with respect to $f$ then it is an isolating neighbourhood with respect to $g$ for $g$ sufficiently close to $f$ in the compact-open topology and the Conley indices with respect to $f$ and $g$ coincide.

Theorem 5.3 (Homotopy property). Assume $f_{s}, s \in[0,1]$, is a family of maps $f_{s}$ : $X \rightarrow X$, continuously depending on $s$. If $N \subset X$ is an isolating neighbourhood with respect to every map $f_{s}$ then $\operatorname{Con}\left(N, f_{s}\right)$ does not depend on $s$.

Theorem 5.4 (Additivity property). Assume the category $\mathcal{C}$ admits the product, denoted $\times$, and $L$ maps products into products. If $N, N_{1}, N_{2}$ are isolating neighbourhoods such that $\operatorname{Inv} N=\operatorname{Inv} N_{1} \cup \operatorname{Inv} N_{2}$, Inv $N_{1} \cap \operatorname{Inv} N_{2}=\emptyset$ then

$$
\operatorname{Con}(N, f)=\operatorname{Con}\left(N_{1}, f\right) \times \operatorname{Con}\left(N_{2}, f\right)
$$

Proof. Put $S_{i}:=\operatorname{Inv} N_{i}$ and select disjoint open neighbourhoods $U_{1}, U_{2}$ of $S_{1}$ and $S_{2}$ respectively. For $i=1,2$ let $M_{i}$ be a compact neighbourhood of $S_{i}$ such that $N_{i} \subset U$. Select $P^{i}$, index pairs for $S_{i}$ in $M_{i}$. One can easily verify that $P:=P^{1} \cup P^{2}$ is an index pair for Inv $N$ in $M:=M_{1} \cup M_{2}$. Obviously $f_{P}=f_{P^{1}} \cup f_{P^{2}}$. Hence, $\left(P_{1} / P_{2},\left[f_{P}\right]\right)$ is the wedge sum of $\left(P_{1}^{1} / P_{2}^{1},\left[f_{P^{1}}\right]\right)$ and $\left(P_{1}^{2} / P_{2}^{2},\left[f_{P^{2}}\right]\right)$. It suffices now to apply $L$.

Theorem 5.5 (Normalization property). The whole space $X$ is an isolating neighbourhood and $\operatorname{Con}(X, f)=L([X],[f])$.

Proof. The conclusion follows from the fact that $(X, \emptyset)$ is an index pair in $X$. 
Theorem 5.6 (Commutativity property; see [4]). Assume $f=\psi \phi, g=\phi \psi$, where $\phi: X \rightarrow Y, \psi: Y \rightarrow X$ are continuous,. If $S \subset X$ is an isolated invariant set with respect to $f$ then $\phi(S)$ is an isolated invariant set with respect to $g$ and $\operatorname{Con}(S, f)=\operatorname{Con}(\phi(S), g)$.

\section{References}

[1] C. C. Conley, Isolated invariant sets and the Morse index, CBMS no. 38, A.M.S., Providence, R.I., 1978.

[2] T. KACZYNski, Conley index for set valued maps: from theory to computation, this volume.

[3] M. Mrozer, The Conley Index on Compact ANR's is of Finite Type, Results in Math. 18 (1990), 306-313.

[4] M. MrozeK, Shape Index and Other Indices of Conley Type for Continuous Maps on Locally Compact Metric Spaces, Fundamenta Mathematicae 145 (1994), 15-37.

[5] M. Mrozek and K. P. Rybakowski, Cohomological Conley Index for Continuous Maps on Metric Spaces, J. Diff. Equ. 90.1 (1991), 143-171.

[6] J. W. Robbin and D. Salamon, Dynamical systems, shape theory and the Conley index, Erg. Th. and Dynam. Sys. 8* (1988), 375-393.

[7] K. P. Rybakowski, On the Homotopy Index for Infinite Dimensional Semiflows, Trans. AMS 269 (1982), 351-382.

[8] A. Szymczak, The Conley index for discrete dynamical systems, Topology and its Applications 66(1995), 215-240. 